\title{
Hadron yields and the phase diagram of strongly interacting matter
}

\author{
M Floris
}

CERN, CH-1211 Geneva 23, Switzerland

\begin{abstract}
This paper presents a brief review of the interpretation of measurements of hadron yields in hadronic interactions within the framework of thermal models, over a broad energy range (from SIS to LHC energies, $\sqrt{s_{\mathrm{NN}}} \simeq 2.5 \mathrm{GeV}-5 \mathrm{TeV}$ ). Recent experimental results and theoretical developments are reported, with an emphasis on topics discussed during the Quark Matter 2014 conference.
\end{abstract}

Keywords: Hadron yields, Thermal model, Statistical Hadronization, LHC, Heavy Ion

\section{Introduction}

It has been known since more than two decades that hadrons in high energy interactions are produced in approximate thermal and chemical equilibrium [1, 2, 3, 4]. The relative abundances of light flavor hadrons are determined by a few thermal parameters and, in the simplest case, approximately proportional to the Boltzmann factors. This is true with the only exception of strange particles, which deviate from the expected equilibrium abundance by a factor which depends on the strangeness content of the particle [5, 6]. In central heavy ion collisions [3], strange particles were however found to follow the expected equilibrium distribution 1

Measurement at different $\sqrt{s}$ revealed that the thermal fit parameters follow a smooth curve in the plane of temperature $T_{\text {ch }}$ and baryo-chemical potential $\mu_{B}$, the so-called "hadron freeze-out curve" [2, 7]. For very high energy collisions, $\mu_{B} \rightarrow 0$ and the temperature extracted from a thermal analysis of the data $\left(T_{\mathrm{ch}} \sim 150-170 \mathrm{MeV}\right)$ is found to be very close to the critical (crossover) temperature estimated in lattice QCD, $T_{\mathrm{c}} \in(143,171) \mathrm{MeV}$ [8]. Despite these observations, the profound meaning of the freeze-out curve [7, 9, 10, 11, 12] remains unclear and several key questions remain unanswered:

- What is the relation of the chemical freeze-out temperature to the QCD critical temperature?

- How is the equilibrium reached?

- What physics mechanisms drive the hadron freeze-out curve?

Recently, higher precision measurements revealed unexpected deviations from the thermal model expectations. The statistical model is an effective model (see e.g. the discussion in [13]) and the small deviations from the equilibrium picture may simply indicate that the precision of the data has become sufficient to reveal its limitations. Their study represents an opportunity for a better understanding of the underlying physics processes.

\footnotetext{
${ }^{1}$ If $4 \pi$ yields are considered instead of mid-rapidity $\mathrm{d} N / \mathrm{d} y$, a strangeness under-saturation factor of about 0.75 is still required at the SPS. Also note that the usage of midrapidity $\mathrm{d} N / \mathrm{d} y$ at low energy is questionable, see for instance the discussion in [1] 3].
} 


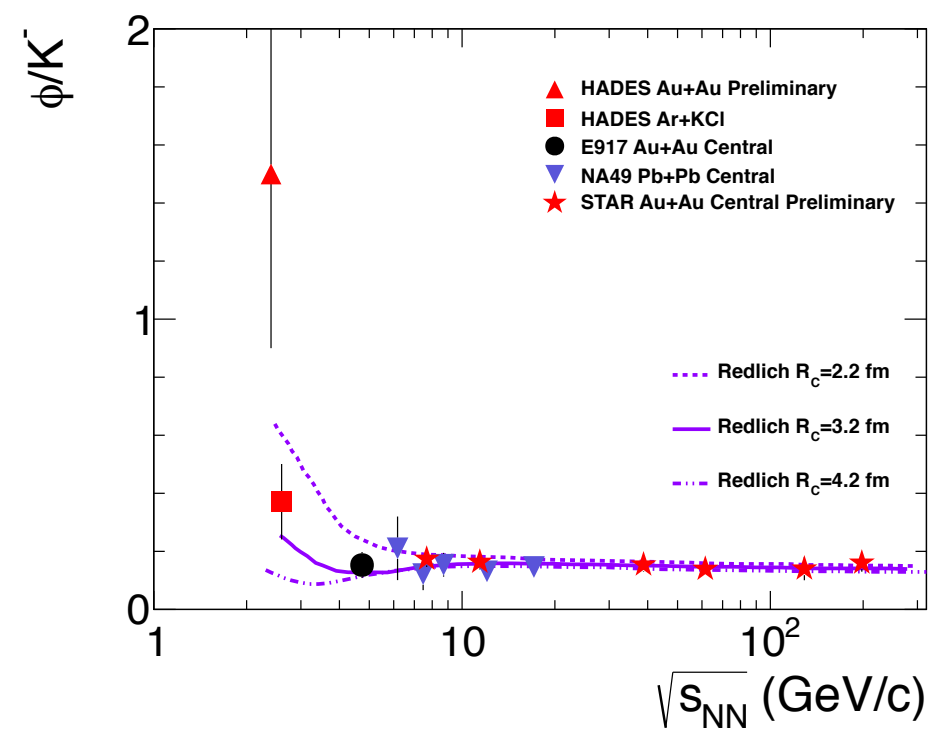

Figure 1. $\phi / \mathrm{K}^{-}$ratio as a function of $\sqrt{s}$ in AA collisions [14].

A qualitative summary of the parameters which control a thermal fit is given below for the non-expert reader. The interested reader should refer e.g. to [1, 3, 4] and references therein for a rigorous discussion.

The main parameters, describing particle production in equilibrium are:

- The temperature $T_{\mathrm{ch}}$ (constrained by ratios of particles with a large mass difference);

- The baryochemical potential $\mu_{B}$ (constrained by anti-baryon/baryon ratios, at LHC it is found $\mu_{B} \sim 0$ );

- The volume V, which acts as a normalization parameter (constrained by the most precisely measured species, typically pions).

Deviations from (grand canonical) equilibrium can be incorporated through empirical under(over)-saturation parameters for strange, charm or light quarks $\left(\gamma_{s}, \gamma_{c}\right.$ and $\left.\gamma_{q}\right)$. The need for $\gamma_{s}$ has already been discussed above. Another approach consist in the implementation of the "canonical suppression" mechanism (i.e. strangeness has to be conserved exactly and not on average) [6] on a smaller volume than the overall size of the system, determined by a "canonical radius" parameter, $R_{C}$. The parameter $\gamma_{c}$ is introduced because charm can only be created in the initial phases of the collisions (it is too heavy to be created thermally) [15] and it is thus expected to be significantly out of equilibrium. While the usage of $\gamma_{s}$ and $\gamma_{c}$ is common to most implementations of the statistical model [3, 16, 17], $\gamma_{q}$ is only found in the non-equilibrium model SHARE [4]. The physical picture in this model is that of an expanding, super-cooled quark-gluon plasma which undergoes a sudden hadronization without further re-interactions. The thermal parameters of the quark-gluon plasma are hence frozen, leading to out-of-equilibrium hadron abundances. From the point of view of the fit, $\gamma_{q}$ allows the relative abundance of mesons and baryons to vary (as it is determined by the number of valence light quarks).

The selection of results presented in this paper reflects the author's bias, especially towards results presented during the Quark Matter 2014 conference. Due to space limitations, the list of references is also incomplete and subject to similar biases: preference is given to reviews and recent papers over the original literature.

\section{Results at low energy}

The HADES collaborations published results on the thermal description of eight particle species $\left(\pi^{-}, \Lambda, \mathrm{K}^{+}, \mathrm{K}_{S}^{0}\right.$, $\phi, \Xi^{-}, \Sigma^{+-}$) in Ar-KCl collisions (approximately 40 nucleons impinging on 40 nucleons) at $\sqrt{s_{\mathrm{NN}}}=2.61 \mathrm{GeV}[18]$. 


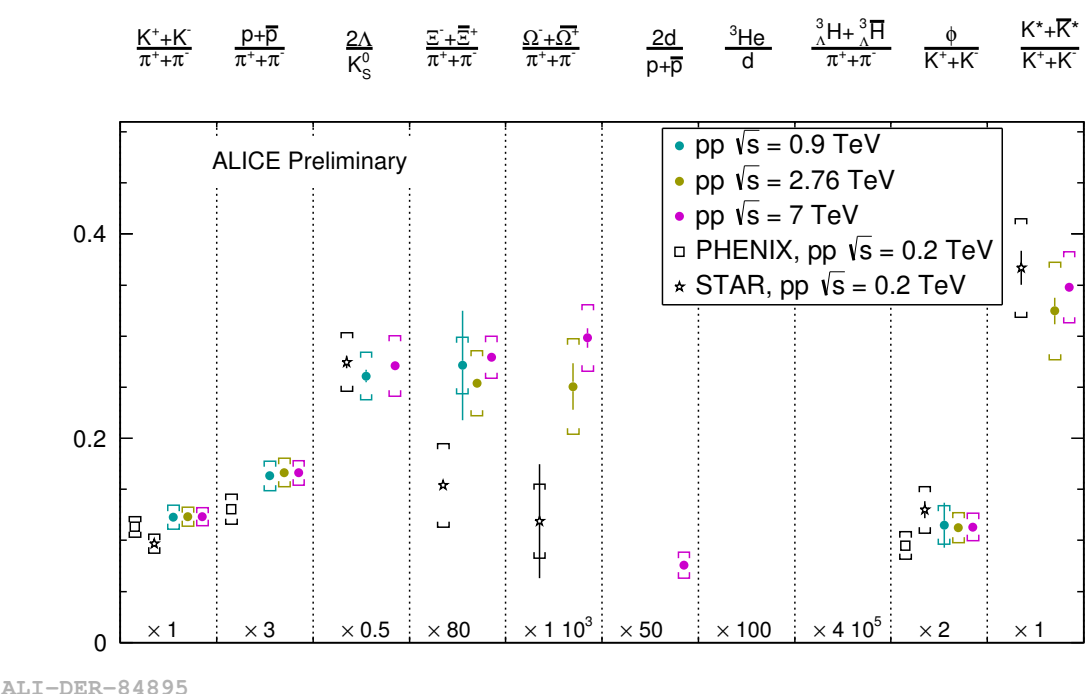

Figure 2. Particle ratios in pp collisions for different $\sqrt{s}$ (the ${ }^{3} \mathrm{He}$ and ${ }_{\Lambda}^{3} \mathrm{H}$ are not yet measured in pp collisions at the LHC. The plot shows two empty columns to allow for an easier visual comparison with the $\mathrm{Pb}-\mathrm{Pb}$ ratios, shown in Fig.4.

These results have been recently complemented by a new fit of four particle ratios $\left(\pi^{-} / p, \mathrm{~K}_{S}^{0} / \Lambda, \mathrm{K}^{-} / \mathrm{K}^{+}, \phi / \mathrm{K}^{-}\right)$ measured in $\mathrm{Au}-\mathrm{Au}$ collisions at $\sqrt{s_{\mathrm{NN}}}=2.4 \mathrm{GeV}$, presented for the first time at this conference [14]. In general, the agreement of these very low energy results with the thermal model calculation is surprisingly good, except for the $\Xi^{-}$ baryon which is underestimated by about an order of magnitude in the model [18]. The $\phi / \mathrm{K}^{-}$ratio shows a hint of an increase at low $\sqrt{s_{\mathrm{NN}}}$ (Fig. 1). This is consistent with the expectation from the canonical suppression mechanism: the $\phi$ meson (being a hidden strangeness particle) is not suppressed, while the $\mathrm{K}$ is, leading to an increase in the $\phi / \mathrm{K}^{-}$ ratio as the energy decreases. This observation is in contrast with results at SPS energies in pp and peripheral $\mathrm{Pb}-\mathrm{Pb}$ collisions, where the canonical suppression mechanism did not describe the $\phi$ data (while the small colliding system size would suggest that it plays an even more important role) [19, 20]. It is also interesting to notice that the $\phi / \mathrm{K}^{-}$ ratio is $\sim 1$ at $\sqrt{s_{\mathrm{NN}}} \sim 2.5 \mathrm{GeV}$, suggesting that about half of the $\mathrm{K}^{-}$originate from $\phi$ decays.

\section{3. pp Collisions at the LHC}

The ALICE collaboration measured the production yield of several particle species in pp collisions at $\sqrt{s}=0.9$, 2.76, and $7 \mathrm{TeV}$. No changes in particle ratios are observed for $\sqrt{s} \gtrsim 1 \mathrm{TeV}$, as shown in Fig. 2 . On the other hand, if the measurements are compared to results at top RHIC energy $(\sqrt{s}=200 \mathrm{GeV})$, significant changes are observed. Most notably, the production of (multi)strange particles relative to pions is seen to increase and then saturate at LHC energies. It was suggested that the grand-canonical limit could be reached in pp collisions at the LHC [21], and this observation would seem to support this idea. However, an actual grand-canonical fit of pp data at the LHC yields a poor quality, with a $\chi^{2} / \mathrm{NDF}>7$ and an increase with system size of the ratio of multistrange baryons to pions is still seen at LHC energies (Fig. 3). The absolute deviations data/model are smaller than 20-30\%. As a final remark, we notice a hint for an increase of the $\mathrm{p} / \pi$ ratio with energy (while an opposite trend is suggested by AA data, sec. 5).

\section{System size dependence at the LHC}

Many new results on identified particle production as a function of charged multiplicity in $\mathrm{p}-\mathrm{Pb}$ collisions at $\sqrt{s_{\mathrm{NN}}}=5.02 \mathrm{TeV}$ were reported by the ALICE collaboration. These studies play an important role in the understanding of hadron production, as they bridge $\mathrm{pp}$ and $\mathrm{Pb}-\mathrm{Pb}$ collisions in terms of multiplicity. In general, the selection of events based on charged particle multiplicity in small systems can introduce non-trivial dynamical biases [22, 23], and results can depend on the choice of the multiplicity estimator. All the results presented in this section were binned 


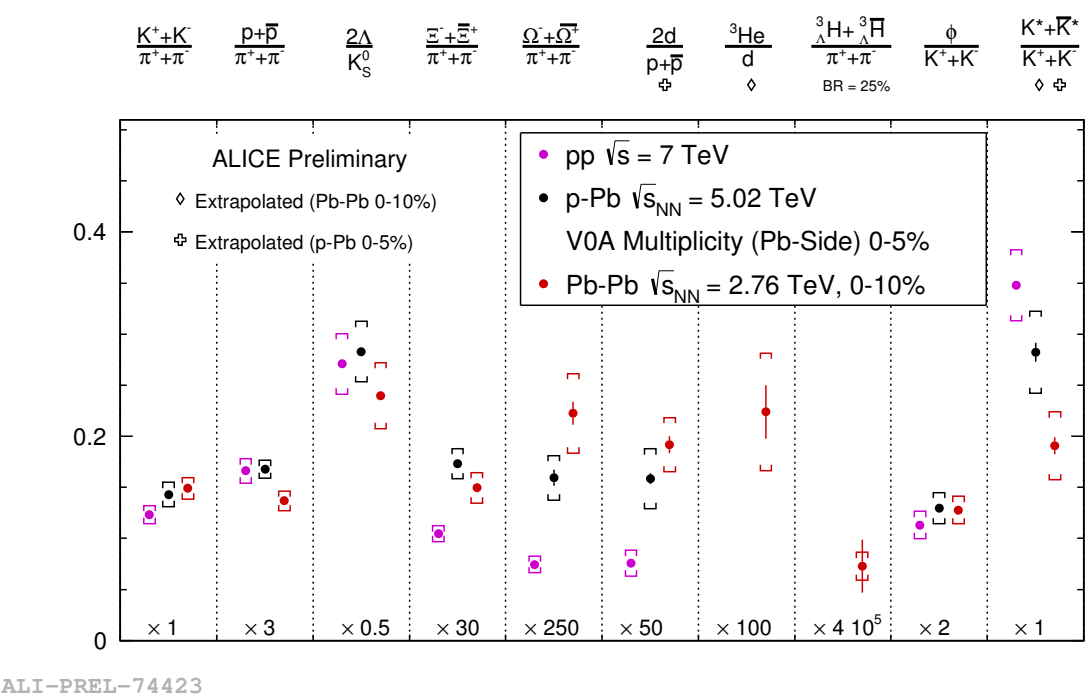

Figure 3. Particle ratios in $\mathrm{pp}, \mathrm{p}-\mathrm{Pb}$, and $\mathrm{Pb}-\mathrm{Pb}$ collisions at $\mathrm{LHC}$ energies.

using quantiles in the amplitude of the ALICE V0A detector, a forward hodoscope covering $2.8<\eta_{\text {lab }}<5.1$ in the lead-going direction [24]. Most of the ratios which change between $\mathrm{pp}$ and $\mathrm{Pb}-\mathrm{Pb}$ collisions attain an intermediate value in $\mathrm{p}-\mathrm{Pb}$ collisions.

(Multi)strange hadrons are seen to increase as a function of multiplicity in $\mathrm{p}-\mathrm{Pb}$ collisions, reaching almost grandcanonical values (with the same temperature $T_{\mathrm{ch}} \simeq 156 \mathrm{MeV}$ observed in $\mathrm{Pb}-\mathrm{Pb}$ collisions), see Fig. 3] and [25]. There is, however, a small tension between the yields of the $\Xi$ and $\Omega$ baryons normalized to pions: the former is higher than the corresponding $\mathrm{Pb}-\mathrm{Pb}$ ratio for the highest multiplicity $\mathrm{p}-\mathrm{Pb}$ collisions, but the latter is below the $\mathrm{Pb}-\mathrm{Pb}$ value. While none of the two is individually significant within uncertainties, their combined effect leads to a large $\chi^{2} / \mathrm{NDF}$ $(\sim 5)$ when a grand-canonical fit of high multiplicity $\mathrm{p}-\mathrm{Pb}$ results is performed. The origin of this tension still needs to be clarified.

The $\mathrm{K}^{*} / \mathrm{K}$ ratio is seen to decrease with increasing multiplicity, as expected in case the $\mathrm{K}^{*}$ decay products undergo elastic scattering in the hadronic medium [26]. The $d / p$ ratio is observed to increase with increasing multiplicity in $\mathrm{p}-\mathrm{Pb}$ collisions, while it is flat as a function of centrality in $\mathrm{Pb}-\mathrm{Pb}$ collisions [27]. This observation constrains the production mechanism of deuterons (thermal production or coalescence models). At this conference, the STAR collaboration reported results on $v_{2}$ of nuclei at RHIC energies, which would support coalescence as the dominant production mechanism [28]. Detailed theoretical modeling as a function of $\sqrt{s_{\mathrm{NN}}}$ would be needed to draw any firm conclusion.

Finally, we notice that there is a hint of a decrease of the baryon/pion ratios $(p / \pi$ and $\Lambda / \pi)$, which would be consistent with the baryon annihilation mechanism proposed to explain particle ratios in central $\mathrm{Pb}-\mathrm{Pb}$ collisions discussed in the next section [29].

\section{Heavy Ion Collisions at High Energy}

Central heavy ion collisions are commonly regarded as the ideal system for the applicability of the thermal model. Equilibrium thermal models were found to give an excellent description of particle yields measured in $\mathrm{Au}-\mathrm{Au}$ collisions at top RHIC energy $\left(\sqrt{s_{\mathrm{NN}}}=200 \mathrm{GeV}, \chi^{2} / \mathrm{NDF} \simeq 1\right.$ when analyzing data from the same experiment, $\chi^{2} / \mathrm{NDF} \sim 2$ when fitting all RHIC results simultaneously [30]). The analysis of data as a function of $\sqrt{s_{\mathrm{NN}}}$, moreover, allowed to extrapolate the parameters to the limiting case of very high energy, which is essentially reached at the LHC [30, 31]. Therefore, the observation of an anomalously low $\mathrm{p} / \pi=0.046 \pm 0.003$ ratio in central collisions at the LHC (a factor $\sim 1.5$ lower then expectations based on $T_{\mathrm{ch}}=164 \mathrm{MeV}$ ) [32, 33] came as a surprise and triggered considerable theoretical discussions. A low value of the $\mathrm{p} / \pi$ ratio is naturally predicted by non-equilibrium models, 


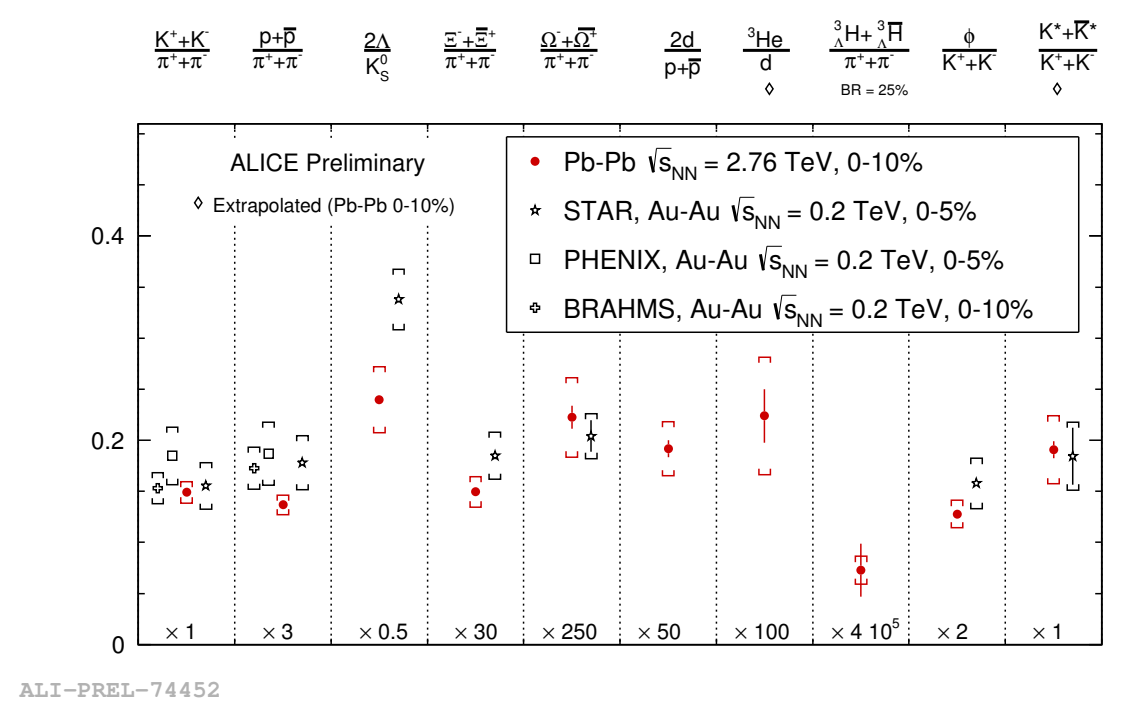

Figure 4. Particle ratios at top RHIC and at LHC energy in AA collisions. In this figure, STAR protons have been corrected for feed-down based on the thermal model estimate.

as a consequence of the lower temperature $\left(T_{\mathrm{ch}} \sim 140 \mathrm{MeV}\right)$ needed to describe the data. The predictions of the $\mathrm{p} / \pi$ ratio with the preferred set of parameters in [34] were indeed in agreement with the data. However, with the same parameters, the $\mathrm{K} / \pi$ ratio was over-estimated by a similarly large factor. The "anomaly" with respect to the equilibrium model expectations is now confirmed by fits which include a complete set of particle species (Fig. 5). It is furthermore observed that the consistency with equilibrium expectations (and extrapolation of RHIC results) is essentially restored if protons and the $\mathrm{K}^{*}$ resonance are excluded from the fit [35].

Given the tension between results at $\sqrt{s_{\mathrm{NN}}}=2.76 \mathrm{TeV}$ and the expectations based on lower energy results, it is natural to ask if there is any change in particle ratios between RHIC and LHC energies, irrespective of any thermal model interpretation. The results are discussed below for $0-10 \%$ central collisions at the LHC [27, 33, 36, 37, 38] and for $0-5 \%$ and $0-10 \%$ results at RHIC [39, 40, 41, 42, 43, 44, 45]. If positive and negative charge states are averaged, the effect of the small charge asymmetry present in mid-rapidity yields at RHIC becomes negligible and particle ratios are expected to be the same at RHIC and LHC. No strong evidence for changes is observed, but the $\mathrm{p} / \pi$ and $\Lambda / \pi$ ratios are found to be lower at the LHC by a factor $\sim 2.3 \sigma$. More recent results from Au-Au collisions at $\sqrt{s_{\mathrm{NN}}}=62.4 \mathrm{GeV}$ and $\sqrt{s_{\mathrm{NN}}}=130 \mathrm{GeV}$ at RHIC show a similar tension between the measured proton yield and equilibrium thermal model calculations [35].

A fit of this extended set of particle species (Fig. 5) with an equilibrium model yields a $\chi^{2} /$ NDF of about 2, which is better than any other colliding system at the LHC, but slightly worse than expected from the fit quality at RHIC. The largest contribution to the $\chi^{2} / \mathrm{NDF}$ comes from the low yield of protons relative to pions. This conclusion persists for the three different thermal model implementations which were used by the ALICE collaboration [4, 16, 30], indicating that the residual differences in those models (minor difference in the hadron list, treatment of charm and of the hadron excluded volume) have a second order effect. The temperature obtained, $T_{\mathrm{ch}}=156 \pm 2 \mathrm{MeV}$, is lower than at RHIC. At this conference, the STAR collaboration reported on high-statistics $\Omega$ measurements in central Au-Au and pp collisions [46]. The measured yield in central $\mathrm{Au}-\mathrm{Au}$ collisions is consistent with the previous result, but lower. This lower yield would be consistent with the chemical freeze-out temperature extracted at the LHC. It will be interesting to study how this affects the thermal fits at RHIC.

A separate discussion should be made for (hyper)nuclei. Having a binding energy much smaller than the estimated temperature at chemical freeze-out, it may be expected that their main production mechanism is not thermal, but they are rather formed by coalescence (sec. 4p. Their inclusion in thermal fits can be questionable. However, it has been argued that a thermal approach is still applicable because hadronic interactions do not change the entropy per baryon and the statistical hadronization and coalescence approaches yield compatible results [47, 48]. The ALICE collab- 


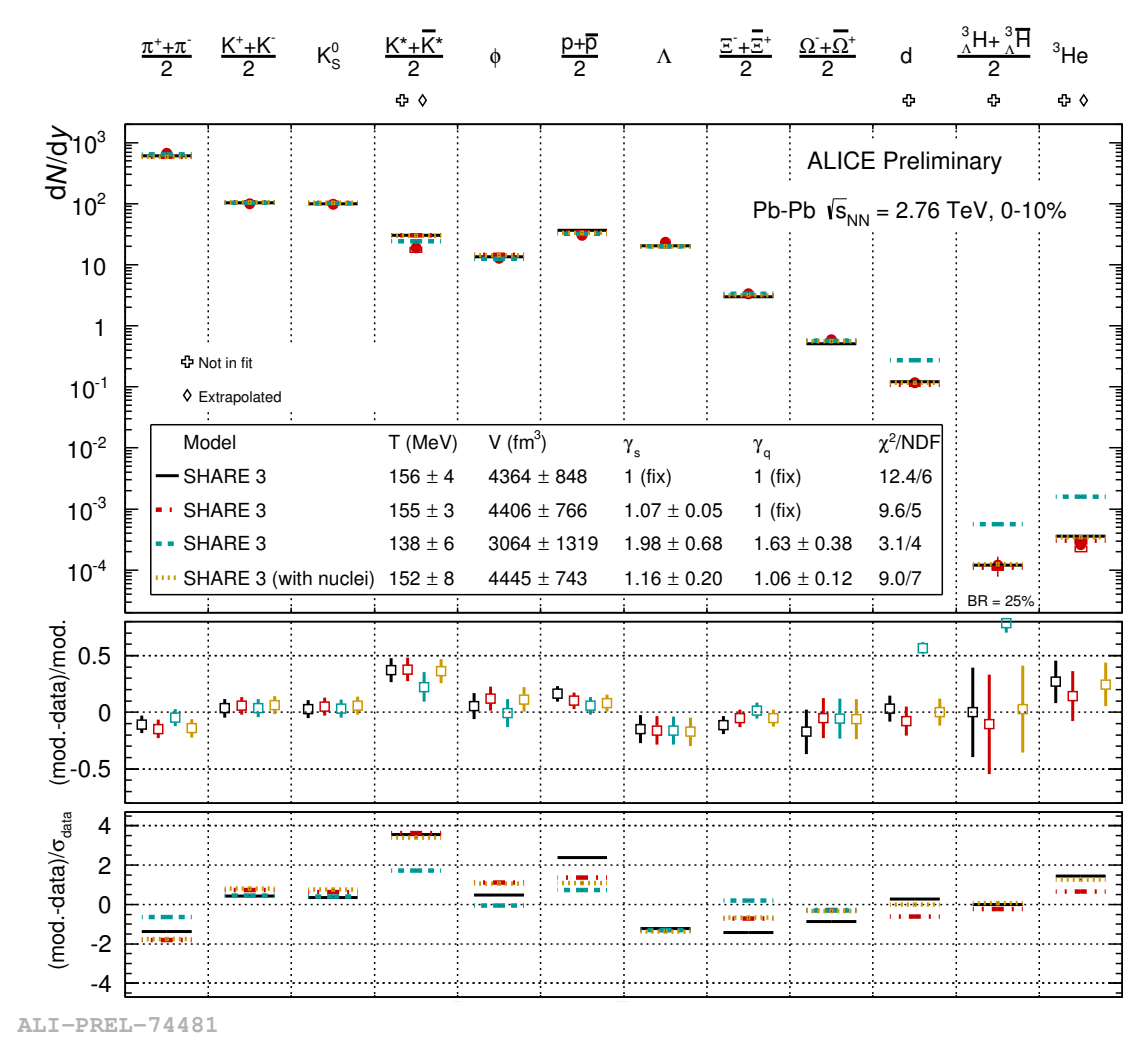

Figure 5. Thermal fit of particle yields in $0-10 \%$ central $\mathrm{Pb}-\mathrm{Pb}$ with the SHARE model for various scenarios.

oration released results on the production of deuterons, ${ }^{3} \mathrm{He}$ and ${ }_{\Lambda}^{3} \mathrm{H}$, found to be in agreement with the equilibrium fits.

Different explanations have been proposed in the literature to explain the particle yields measured at the LHC and the "anomaly" in the $\mathrm{p} / \pi$ ratio, as summarized below.

Incomplete Hadron List. One of the basic ingredients in the thermal models is the list of hadrons and high mass resonances which feed-down to the (stable) measured species. It is known that this list is incomplete, and it was argued (see e.g. [48]) that this could explain the tension, as decays of high mass resonances would affect pions more than protons. Quantitative calculations were made in [49, 50, 51], where it was shown that reasonable assumptions on high mass resonances based on the Hagedorn spectrum could explain the low $\mathrm{p} / \pi$. However, these additional states could potentially spoil the agreement with other particle ratios (most notably, multistrange baryons, which could however be included as discussed [52]) and some of the underlying assumptions of the model are not constrained by first principles.

Non-Equilibrium Thermal Model. In the framework of the non-equilibrium thermal model, as implemented in the SHARE code [4], it is possible to find a set of parameters which describes all hadrons except nuclei, with a very good $\chi^{2} / \mathrm{NDF}$ [4, 53]. An interesting feature of this description is that the physical parameters of the fireball at freezeout (pressure, energy density and entropy density) are rather constant as a function of energy and centrality [53], consistently with the physical picture underlying this model. Additional support for this picture comes from the combined study of yields and transverse momentum distributions discussed in [54, 55]. The main weak points of the non-equilibrium fits come from the additional free parameters and the relatively small number of particles included in the fits.

In Fig. 5. SHARE is used both in the equilibrium and in the non-equilibrium mode ( $\gamma_{q}$ fixed to unity or free) in order to fit the ALICE measurement, including or not nuclei. It the equilibrium model, nuclei follow the systematics 
established by other particles, as discussed above. The non-equilibrium model, on the other hand, overestimates nuclei production by a large factor. If the nuclei are included in the fit, the $\gamma_{q}$ parameter converges to unity and the fit quality degrades, with deviations similar to the equilibrium fits.

Hadronic interactions. Inelastic processes in the hadronic phase may not be completely negligible as normally assumed and could affect in a stronger way baryons than mesons. In particular, it was suggested that baryons annihilation in the hadronic phase could have a major role in the reduction of the proton yields. These effects have been studied quantitatively in [29, 56] using the UrQMD hadron transport code. They have been found to be consistent with the data. The main criticism to this approach is the fact that reverse reactions with more than 2 bodies are so far not implemented in UrQMD, even if it has been argued that they will not suppress the effect of the annihilation (see also [57]).

Flavor hierarchy at freeze-out. Since the transition from the deconfined medium to hadronic matter is a smooth crossover, the freeze-out temperature cannot be uniquely identified but it depends on the observable under study. It was further suggested in [58], based on lattice and Hadron Resonance Gas (HRG) calculations, that the (crossover) transition temperature for light and strange quarks may be different ( $150 \mathrm{MeV}$ and $165 \mathrm{MeV}$, respectively). This would subsequently lead to a separate freeze-out at slightly lower temperatures. In this picture, a single temperature should not describe all data, explaining the tensions observed in the thermal model fits. The lattice and HRG calculations can be directly confronted with the experiments using higher moments of conserved charges (net charge, net baryon number, net strangeness). Recently, the STAR collaboration measured the higher order moments of the net charge and net proton distribution [59,60] as a function of beam energy. These have been compared with theoretical calculations in [61, 62] to extract a light flavor freeze-out temperature and baryochemical potential, which were then used to compute particle ratios in [62, 63]. The ratios of light flavor particles are found to be consistent with these parameters, while strange particles are underestimated, consistent with the expectation. In this comparison there are however important caveats related to the limitations of the HRG models, to the limited acceptance of the detectors and to the fact that the experiments measure net protons and not the real conserved charge (net baryon). A key crosscheck would be a measurement of net-strangeness, but this may not be easily attainable as it requires the measurement of several multistrange baryons.

Outlook. At least four different mechanisms have been proposed to explain the yields measured at the LHC, leading to a significant spread in the estimated chemical freeze-out temperature ( $T_{\text {ch }}$ in the range $\left.130-165 \mathrm{MeV}\right)$. It is not yet established which is the correct mechanisms and how this temperature relates to the QCD phase transition temperature.

Additional experimental constraints could help to identify the correct mechanism, in particular:

- Precise measurements of the trends of particle ratios as a function of centrality (to constrain the effect of the hadron phase, expected to be strongly centrality dependent; current measurements at the LHC do not show significant changes within uncertainties for most particle ratios);

- Measurement of "heavy" light-flavor mesons (for instance, the expectations for the $\omega$ meson in the equilibrium and non-equilibrium models differ by $\sim 1.4$ );

- Measurements at lower energy with the improved vertex detectors (this would reduce some uncertainties, for instance feed-down uncertainties in the proton measurements);

- Additional higher moment measurements at LHC and RHIC, in particular net strangeness (to constrain the freezeout temperature of strange particles in the hierarchical freezeout scenario).

\section{Acknowledgments}

I am indebted to many colleagues for the stimulating discussion, for the precious suggestions and for providing me with the experimental data and theoretical calculations: P. Alba, F. Becattini, J. Noronha-Hostler, M. Petrán, C. Ratti, K. Redlich; the colleagues from the STAR and HADES collaborations, in particular L. Kumar, M. Lorenz, N. Xu, X. Zhu; my colleagues from the ALICE collaboration, in particular A. Andronic, F. Antinori, R. Bellwied, B. Dönigus, B. Hippolyte, A. Kalweit, H. Oeschler, M. Ploskon, R. Preghenella, K. Safarik, J. Schukraft. 


\section{References}

[1] F. Becattini, R. Fries. in "Relativistic heavy ion physics", Landolt-Boernstein 1-23. arXiv:0907.1031

[2] J. Cleymans, K. Redlich, Phys.Rev.Lett. 81 (1998) 5284-5286. arXiv: nucl-th/9808030

[3] P. Braun-Munzinger, K. Redlich, J. Stache arXiv: nucl-th/0304013

[4] M. Petran, J. Letessier, V. Petracek, J. Rafelski, Phys.Rev. C88 (3) (2013) 034907. arXiv:1303.2098

[5] J. Rafelski, B. Muller, Phys.Rev.Lett. 48 (1982) 1066.

[6] A. Tounsi, K. Redlich arXiv:hep-ph/0111159

[7] J. Cleymans, H. Oeschler, K. Redlich, S. Wheaton, Phys.Rev. C73 (2006) 034905. arXiv:hep-ph/0511094

[8] S. Borsanyi, et al., JHEP 1009 (2010) 073. $\sim \operatorname{arXiv:1005.3508}$

[9] R. Stock, Phys.Lett. B456 (1999) 277-282. arXiv: hep-ph/9905247

[10] P. Braun-Munzinger, J. Stachel, J.Phys. G28 (2002) 1971-1976. arXiv: nucl-th/0112051|

[11] P. Braun-Munzinger, J. Stachel, C. Wetterich, Phys.Lett. B596 (2004) 61-69. arXiv: nucl-th/0311005

[12] A. Andronic, D. Blaschke, P. Braun-Munzinger, J. Cleymans, K. Fukushima, et al., Nucl.Phys. A837 (2010) 65-86. arXiv:0911.4806

[13] F. Becattini, P. Castorina, A. Milov, H. Satz, Eur.Phys.J. C66 (2010) 377-386. arXiv:0911.3026

[14] M. Lorenz, et al., in: These Proceedings, 2014.

[15] P. Braun-Munzinger, J. Stachel, Phys.Lett. B490 (2000) 196-202. arXiv: nucl-th/0007059

[16] S. Wheaton, J. Cleymans, Comput.Phys.Commun. 180 (2009) 84-106. arXiv: hep-ph/0407174

[17] F. Becattin arXiv:0901.3643

[18] G. Agakishiev, et al., Eur.Phys.J. A47 (2011) 21. arXiv: 1010.1675

[19] I. Kraus, J. Cleymans, H. Oeschler, K. Redlich, Phys.Rev. C79 (2009) 014901. arXiv:0808.0611

[20] F. Becattini, J. Manninen, J.Phys. G35 (2008) 104013. arXiv: 0805.0098

[21] F. Becattini, P. Castorina, A. Milov, H. Satz, J.Phys. G38 (2011) 025002. arXiv: 0912.2855

[22] A. Toia, et al., in: These Proceedings, 2014.

[23] A. Adare, et al arXiv:1310.4793

[24] B. Abelev, et al $\sim \operatorname{arXiv:1402.4476}$

[25] D. Alexandre, et al., in: These Proceedings, 2014.

[26] F. Bellini, et al., in: These Proceedings, 2014.

[27] N. Martin, et al., in: These Proceedings, 2014

[28] R. Haque, et al., in: These Proceedings, 2014.

[29] F. Becattini, E. Grossi, M. Bleicher, J. Steinheimer, R. Stock $\sim \operatorname{arXiv:1405.0710}$

[30] A. Andronic, P. Braun-Munzinger, J. Stachel, Phys.Lett. B673 (2009) 142-145. arXiv:0812.1186

[31] J. Cleymans, I. Kraus, H. Oeschler, K. Redlich, S. Wheaton, Phys.Rev. C74 (2006) 034903. arXiv: hep-ph/0604237

[32] B. Abelev, et al., Phys.Rev.Lett. 109 (2012) 252301. arXiv:1208.1974

[33] B. Abelev, et al., Phys.Rev. C88 (4) (2013) 044910. arXiv:1303.0737

[34] J. Rafelski, J. Letessier, Phys.Rev. C83 (2011) 054909.

[35] A. Andronic, P. Braun-Munzinger, K. Redlich, J. Stachel, Nucl.Phys. A904-905 (2013) 535c-538c. arXiv: 1210.7724

[36] B. Abelev, et al., Phys.Lett. B728 (2014) 216-227. arXiv: 1307.5543

[37] B. Abelev, et al., Phys.Rev.Lett. 111 (22) (2013) 222301. arXiv:1307.5530

[38] B. Abelev, et al $\sim$ arXiv: 1404.0495

[39] B. Abelev, et al., Phys.Rev. C79 (2009) 034909. arXiv:0808.2041

[40] I. Arsene, et al., Phys.Rev. C72 (2005) 014908. arXiv: nucl-ex/0503010

[41] S. Adler, et al., Phys.Rev. C69 (2004) 034909. arXiv: nucl-ex/0307022

[42] J. Adams, et al., Phys.Rev.Lett. 98 (2007) 062301. $\sim \operatorname{arXiv:nucl-ex/0606014}$

[43] M. Aggarwal, et al., Phys.Rev. C84 (2011) 034909. arXiv: 1006.1961

[44] B. Abelev, et al., Phys.Rev. C79 (2009) 064903. arXiv:0809.4737

[45] G. Agakishiev, et al., Phys.Rev.Lett. 108 (2012) 072301. arXiv: 1107.2955

[46] X. Zhu, et al., in: These Proceedings, 2014.

[47] P. Siemens, J. I. Kapusta, Phys.Rev.Lett. 43 (1979) 1486-1489.

[48] J. Stachel, A. Andronic, P. Braun-Munzinger, K. Redlich, J.Phys.Conf.Ser. 509 (2014) 012019. arXiv: 1311.4662

[49] J. Noronha-Hostler, C. Greiner, I. Shovkovy, Phys.Rev.Lett. 100 (2008) 252301. arXiv:0711.0930

[50] J. Noronha-Hostler, C. Greiner arXiv: 1405.7298

[51] J. Noronha-Hostler, et al., in: These Proceedings, 2014.

[52] J. Noronha-Hostler, M. Beitel, C. Greiner, I. Shovkovy, Phys.Rev. C81 (2010) 054909. arXiv:0909.2908

[53] J. Rafelski, M. Petran, Acta Phys.Polon.Supp. 7 (1) (2014) 35-47. arXiv:1403.4036

[54] V. Begun, W. Florkowski, M. Rybczynsk $\sim \operatorname{arXiv:1312.1487}$

[55] V. Begun, W. Florkowski, M. Rybczynsk $\sim \operatorname{arXiv:1405.7252}$

[56] F. Becattini, M. Bleicher, T. Kollegger, T. Schuster, J. Steinheimer, et al., Phys.Rev.Lett. 111 (2013) 082302. arXiv:1212.2431

[57] Y. Pan, S. Prat $\sim \operatorname{arXiv:1210.1577}$

[58] R. Bellwied, S. Borsanyi, Z. Fodor, S. D. Katz, C. Ratti, Phys.Rev.Lett. 111 (2013) 202302. arXiv: 1305.6297

[59] L. Adamczyk, et al arXiv:1402.1558

[60] L. Adamczyk, et al., Phys.Rev.Lett. 112 (2014) 032302. arXiv:1309.5681

[61] S. Borsanyi, Z. Fodor, S. Katz, S. Krieg, C. Ratti, et al. (and these proceedings). arXiv:1403.4576

[62] P. Alba, W. Alberico, R. Bellwied, M. Bluhm, V. M. Sarti, et al arXiv:1403.4903

[63] M. Bluhm, et al., in: These Proceedings, 2014. 